\title{
Helicobacter pylori CagA promotes epithelial mesenchymal transition in gastric carcinogenesis via triggering oncogenic YAP pathway
}

Nianshuang $\mathrm{Li}^{1,2}$, Yan Feng ${ }^{2}, \mathrm{Yi} \mathrm{Hu}^{1}$, Cong He${ }^{1}$, Chuan Xie', Yaobin Ouyang ${ }^{1}$, Stephen C. Artim², Deqiang Huang ${ }^{1}$, Yin Zhu', Zhijun Luo ${ }^{3}$, Zhongming Ge $\mathrm{C}^{2^{*+}}$ and Nonghua Lu ${ }^{1 *+}$

\begin{abstract}
Background: Helicobacter pylori (H. pylori) delivers oncoprotein CagA into gastric epithelial cells via the T4SS and drives activation of multiple oncogenic signalling pathways. YAP, a core effector of the Hippo tumour suppressor pathway, is frequently overexpressed in human cancers, suggesting its potential tumor-promoting role. Although CagA is a casual factor in H. pylori induced gastric carcinogenesis, the link between CagA and YAP pathway has not been identified. In this work, we investigated the regulation of oncogenic YAP pathway by H. pylori CagA.

Methods: Expression of YAP and E-cadherin protein in human gastric biopsies were assessed by immunohistochemistry. H. pylori PMSS1 cag $A^{-}$isogenic mutant strains were generated. Gastric epithelial cells were co-cultured with $\mathrm{H}$. pylori wild-type cag $A^{+}$strains or isogenic mutants and were also treated by recombinant CagA expression. Immunofluorescence was performed for YAP localization. Immunoblot and quantitative PCR were performed for examining levels of YAP, downstream effectors and markers of epithelial-mesenchymal transition. Verteporfin and siRNA silencing were used to inhibit YAP activity.
\end{abstract}

Results: YAP is significantly upregulated in human gastric carcinogenesis. We generated PMSS1 CagA isogenic mutant strains with chloramphenicol resistance successfully. Our analysis indicated that $H$. pylori infection induced YAP and downstream effectors in gastric epithelial cells. Importantly, knockout of CagA in 7.13 and PMSS1 strains reduced the expression of YAP by H. pylori infection. Moreover, Inhibition of YAP suppressed H. pylori infection-induced Epithelialmesenchymal transition (EMT).

Conclusion: Our results indicated that H. pylori CagA as a pathogenic protein promotes oncogenic YAP pathway, which contributes to EMT and gastric tumorigenesis. This study provided a novel mechanistic insight into why cag $A^{+}$ H. pylori infection is associated with a higher risk for the development of gastric cancer.

Keywords: H. pylori CagA, YAP, Epithelial-mesenchymal transition, Gastric carcinogenesis

\footnotetext{
*Correspondence: zge@mit.edu; lunonghua@ncu.edu.cn

†Zhongming Ge and Nonghua Lu contributed equally to this work.

${ }^{2}$ Division of Comparative Medicine, Massachusetts Institute of Technology, 77

Massachusetts Avenue, Cambridge, MA 02139, USA

'Department of Gastroenterology, The First Affiliated Hospital of Nanchang

University, 17 Yong Waizheng Street, Donghu District, Nanchang 330006,

Jiangxi Province, China

Full list of author information is available at the end of the article
}

C The Author(s). 2018 Open Access This article is distributed under the terms of the Creative Commons Attribution 4.0 International License (http://creativecommons.org/licenses/by/4.0/), which permits unrestricted use, distribution, and reproduction in any medium, provided you give appropriate credit to the original author(s) and the source, provide a link to the Creative Commons license, and indicate if changes were made. The Creative Commons Public Domain Dedication waiver (http://creativecommons.org/publicdomain/zero/1.0/) applies to the data made available in this article, unless otherwise stated. 


\section{Introduction}

The gram-negative microaerophilic bacterium, Helicobacter pylori (H. pylori), infects approximately half of the world's population and is mostly acquired in childhood [1, 2]. It has been estimated that $80-90 \%$ of $H$. pylori infection is asymptomatic; $10-15 \%$ and $1-3 \%$ of $H$. pylori-infected individuals develop gastric ulcer and gastric adenocarcinoma respectively [3]. H. pylori infection can lead to gastric carcinogenesis through the histopathological Correa cascade of steps which include atrophic chronic gastritis, intestinal metaplasia and dysplasia culminating in gastric cancer [4]. The clinical outcome of $H$. pylori infection is determined by multiple factors including pathogenicity of individual $H$. pylori strains, host susceptibility and environmental stimuli $[5,6]$. Epidemiological data indicate that infection with $\operatorname{cagA}+$ (cytotoxin-associated gene A) H. pylori strains is associated with more severe gastric inflammation and a higher risk for the development of pre-neoplastic lesions including intestinal metaplasia and dysplasia in comparison with cagA- $H$. pylori strains [7]. It has been also documented that CagA plays an important role in $H$. pylori-induced gastric tumorigenesis in rodent models $[8,9]$. CagA is encoded on the cag pathogenicity island and can be delivered into gastric epithelial cells through Type IV secretion system [10]. Upon delivery into the target cells, CagA promotes various pro-oncogenic signalling pathways [11]. Notably, CagA in the host cells is tyrosine-phosphorylated and interacts with SHP-2, leading to cellular morphological changes associated with increased cell motility and scattering, termed the "hummingbird phenotype" $[12,13]$.

The mammalian Hippo tumor suppressor signalling pathway is crucial in maintaining developmental organ size and tissue homeostasis [14]. The central components of the Hippo pathway comprise MOB1 (Mps One Binder kinase activator), Sav1 (also known as WW45), MST1/2 (STE20-like protein kinase 1), LATS1/2 (large tumor suppressor 1) and two major downstream effectors YAP (Yes-Associated Protein), and transcriptional co-activator TAZ (PDZ-binding motif) [15]. Canonically, when this signalling is on, MST1/2 phosphorylates LATS1/2 at Thr1079/Thr1041 sites, stimulated by SAV1 and MOB1. Then phosphorylated LATS1 directly phosphorylates YAP at Ser127, resulting in cytoplasmic sequestration via binding to $14-3-3$ proteins. By contrast, when Hippo signalling is off, YAP is activated and translocated from cytoplasm into nucleus through interaction with transcriptional factors TEADs $[16,17]$. This process leads to expression of downstream oncoproteins, such as connective tissue growth factor $(C T G F)$, cysteine-rich angiogenic inducer 61 (CYR61) and MYC. Increased expression of YAP is positively associated with progression of different human cancers [18]. Inducible overexpression of YAP in mouse liver can lead to expansion of liver size and eventual hepatocellular carcinoma [19]. Activation of
YAP is functionally important for proliferative and pro-survival activity in colon cancer cell lines [20]. Therefore, YAP is considered an oncogenic protein.

Epithelial mesenchymal transition (EMT), a hallmark of tumorigenic transformation, is a cellular program by which epithelial cells lose cell-cell adhesion and acquire mesenchymal traits [21][. During this process, epithelial cells undergo marked biochemical changes, including enhanced cell elongation, loss of polarity, and migratory capacity. Expression of some common biomarkers involving cellular proliferation, including suppression of epithelial markers (e.g. E-cadherin, ZO-1) and upregulation of mesenchymal markers (e.g. N-cadherin, Vimentin, Snail and Slug), can promote EMT [22]. It has been experimentally documented that overexpression of YAP results in reduction of the epithelial marker E-cadherin and phenotypic alteration that is associated with EMT, promoting cancer cell invasion and metastasis [23, 24].

Additionally, it has been reported that intracellular CagA can disrupt cell-cell junctions and cause loss of epithelial adhesion, thereby directly activating EMT [25]. Moreover, a recent study showed that YAP mRNA levels were significantly elevated by $H$. pylori SS1 infection in C57BL/6 mice in combination with administration of 1-methyl-3-nitro-1-nitrosoguanidine (MNNG) [26]. However, the role of CagA in $H$. pylori-induced activation of YAP signalling pathway is poorly understood. In this study, by using $\mathrm{CagA}^{+}$H. pylori strains PMSS1 and 7.13 as well as their $\triangle$ cagA mutants, we characterized effects of CagA on activation of the YAP signalling pathway in AGS cells. Also, we examined how $H$. pylori infection in humans influenced expression of YAP and an epithelial marker E-cadherin during progression of a cascade of gastric cancer from chronic non-atrophic gastritis (CNAG), intestinal metaplasia (IM), dysplasia (Dys) to cancer (GC).

\section{Materials and methods \\ Antibodies, siRNA and plasmids}

Antibodies and their sources were as follows: YAP inhibitor verteporfin from Sigma-Aldrich (St. Louis, MO, USA) for western blot assay, Anti-GAPDH (\#2118), Anti-YAP (\#4912), Anti-Phospho-YAP Ser127 (\#4911), and Anti-Slug (\#9585), from Cell Signaling Technology (Beverly, MA, USA); Anti-TAZ (HPA007415) from Sigma (St. Louis, MO, USA) (Anti-E-cadherin (\#610405), Anti-N-cadherin (\#610921) from BD Biosciences (San Jose, CA USA); Anti-CagA (sc-28,368) and Anti-phospho-tyrosine (sc-7020) from Santa Cruz Biotechnology (Santa Cruz, CA, USA); anti-H. pylori urease B (ab127916) from Abcam (Cambridge, MA, USA). For immunohistochemistry assay, Anti-YAP (\#4912) from Cell Signaling Technology, Anti-TAZ (HPA007415) from Sigma, Anti-E-cadherin (\#610405) from BD Biosciences For immunofluorescence assay, Anti-YAP (\#4912) from Cell Signaling Technology, 4',6-diamidino-2-phenylindole (DAPI), 
fluorescein isothiocyanate (FITC)-conjugated donkey antirabbit antibodies from Invitrogen (Thermo Fisher Scientific, Suwanee, GA, USA). The recombinant plasmid of YAP CDNA, CagA were constructed and purchased from GeneChem, Shanghai, China. YAP siRNA was purchase from Santa Cruz Biotechnology.

\section{H. pylori strains and generation of PMSS1 $\triangle c a g A$ isogenic mutants}

$\mathrm{CagA}^{+}$H. pylori strain PMSS1 (pre-mouse Sydney strain 1) and its mouse-adapted SS1 which is deficient in CagA function due to a mutation in $\operatorname{cag} Y$ were used in this study. $H$. pylori strain 7.13 and an isogenic cagA mutant were also included in this study, which were kindly provided by Dr. Richard Peek at Vanderbilt University Medical Center, Nashville, TN, USA. All H. pylori strains were cultured on trypticase soy agar with $5 \%$ sheep blood agar plates (Thermo Fisher Scientific) for in vitro passage.

For constructing PMSS1 $\triangle$ cagA isogenic mutants, an overlapping PCR amplicon (namely cagAdel) consisting of the upstream and downstream regions of the PMSS1 cagA gene(s) were produced using a pair of primers cagAupF and $\operatorname{cag} A \mathrm{~L}-5^{\prime} \mathrm{RXS}$ or $\operatorname{cag} A \mathrm{dnR}$ and $\operatorname{cagAF}-3^{\prime} \mathrm{FXS}$ respectively as described previously (Additional file 3 : Fig. S3A). Subsequently, a 0.7-kb cat (chloramphenicol acetyltransferease) cassette was ligated into a SmaI site in the overlapping site of cagAdel; two recombinant plasmids 54 and 55 which had the opposite orientations in reference to that of $\operatorname{cag} A$ were selected (Additional file 3: Fig. S3B and 4C). Then these recombinant plasmids were introduced into PMSS1 strain by electroporation or natural transformation as described previously [27]. Transformants were screened on sheep blood agar containing $25 \mu \mathrm{g} / \mathrm{ml}$ of chloramphenicol; four $\mathrm{Cm}^{\mathrm{R}}$ transformants, namely 54E, 54 $\mathrm{N}, 55 \mathrm{E}$ and $55 \mathrm{~N}$, were obtained. Complete deletion of $\operatorname{cagA}$ from the genomes of these transformants were verified using PCR with four primer sets F1/R1 and F2/R2 for targeting cagA, and Fup $/ 54$ and Fup/55 for detecting the locus containing the sequence upstream of cagA and the cat cassette as well as direct sequencing. The sequences of all primers were listed in Additional file 6: Table S3.

\section{Cell culture and cell transfection}

Human gastric epithelial cells AGS and MKN-45 (CRL-1739; ATCC, Manassas, VA, USA) were cultured in RPMI 1640 (Gibco, CA, USA) containing 10\% fetal bovine serum (Sigma Aldrich, MO, USA) and 1\% penicillin/streptomycin (Gibco) at $37^{\circ} \mathrm{C}$ in $5 \% \mathrm{CO}_{2}$ atmosphere. AGS cells were transiently transfected with the YAP CDNA plasmid using Lipofectamine 2000 (Invitrogen) according to the manufacturer's instructions.

When the cells reached $70 \%$ confluence, they were serum-starved overnight prior to $H$. pylori infection. All $H$. pylori strains were grown in Brucella broth supplemented with $5 \%$ fetal bovine serum at $37^{\circ} \mathrm{C}$ for $24 \mathrm{~h}$ under microaerobic conditions; $\mathrm{OD}_{600 \mathrm{~nm}}$ of bacterial suspensions was then adjusted with 1\% FBS DMEM media to concentrations corresponding to a multiplicity of infection (MOI; the number of bacteria per cell at the onset of infection) of 50, 100 and 200. After co-culture with H. pylori strains for 6 or $24 \mathrm{~h}$, AGS cells were collected for qPCR, Western blotting, or immunofluorescent staining.

\section{Western blotting}

Cells were treated with $H$. pylori strains or in combination with verteporfin. Cells were washed three times in cold PBS before adding cell lysis buffer (Cell signaling, Beverly, MA, USA) with protease inhibitor cocktail (Roche, Amherst, CA, USA). Protein concentration was determined using a BCA assays. The cell lysates with an equal amount of protein $(25 \mu \mathrm{g})$ was separated on $10 \%$ SDS-PAGE and transferred to nitrocellulose membranes. The membranes were blocked in Odyssey blocking buffer (Li-COR, Lincoln, NE, USA) at room temperature for $1 \mathrm{~h}$ and then incubated with primary antibodies in $5 \%$ BSA-TBST at $4{ }^{\circ} \mathrm{C}$ overnight. The membranes were then incubated with IRDye-conjugated anti-mouse and anti-rabbit secondary antibodies (1:10000, Li-COR) in Odyssey blocking buffer containing $0.2 \%$ Tween-20 at room temperature for $1 \mathrm{~h}$. The protein bands on the nitrocellulose blots were imaged and quantified by the Odyssey imaging system through the analyze module (Li-COR). Band intensity of proteins of interest was normalized to GAPDH.

\section{Real-time quantitative PCR analysis}

For measuring mRNA levels of target genes, total RNA was extracted using Trizol Reagents (Invitrogen) and converted to cDNA using the High Capacity cDNA Archive kit (Thermo Fisher Scientific) according to the manufacturer's instructions. Levels of YAP, CTGF and CYR61 mRNA were measured by qPCR using commercial probe mixtures ((Thermo Fisher Scientific) in the 7500 Fast Real-Time PCR system (Life Technologies). Taqman Fast Universal PCR Master Mix (Thermo Fisher Scientific) was used in this assay. Transcript levels were normalized to the endogenous control glyceraldehyde-3-phosphate dehydrogenase mRNA $(G A P D H)$ and expressed as fold change compared with sham-dosed control mice using the Comparative $C_{T}$ method (Applied Biosystems User Bulletin no. 2).

\section{Cell elongation assay}

AGS cells were seeded onto 12-well cell culture plates at a density of $1 \times 10^{5}$ cells per well. After incubating for $3 \sim 4 \mathrm{~h}, \mathrm{H}$. pylori cells were added at AGS cells at MOI of 200 . At $24 \mathrm{~h}$ post infection, cells were stained by a three-step staining set (Thermo Fisher Scientific). Images were captured under a contrast microscope (Zeiss Axioskop 
2). The length and breadth for at least 30 elongated cells each group were measured using ImageJ software, and then the length-to-breadth ratios were statistically analyzed.

\section{Immunofluorescence}

Cells were washed three times with iced-cold PBS and incubated for $15 \mathrm{~min}$ at room temperature with $4 \%$ formaldehyde in PBS. The cells were permeabilized with $0.25 \%$ Triton X-100 for $15 \mathrm{~min}$ and then blocked for $1 \mathrm{~h}$ in PBS with 3\% bovine serum albumin (BSA). The cells were incubated with primary antibody of YAP or E-cadherin overnight at $4{ }^{\circ} \mathrm{C}$, and then incubation with anti-rabbit-FITC or Alex-Fluor-568-conjugated anti-mouse secondary antibody. Cell nuclei were counter-stained with DAPI. All slides were examined, and images were captured using a fluorescent microscope (Zeiss Axioskop 2).

\section{Cell migration and invasion assay}

For Boyden chamber assay, AGS cells were suspended in $200 \mu \mathrm{l}$ serum-free DMEM and seeded into a 24-well Boyden chamber $(8 \mu \mathrm{m}$ pore size, Corning, NY, USA) with Matrigel-pre-coated inserts (BD, Franklin Lakes, NJ, USA). The chambers were incubated in DMEM medium with $10 \%$ FBS. After a $24 \mathrm{~h}$ incubation, cells attached to the chambers' lower surface were fixed with $4 \%$ paraformaldehyde, and then stained with $0.1 \%$ crystal violet, and counted under a microscope (Nikon Ti-S). Wound healing assay and transwell assay for cell migration and invasion were performed as previously described [28].

\section{Gastric specimens and immunohistochemistry}

A total of 199 paraffin-embedded human gastric adenocarcinoma and corresponding non-cancerous specimens were obtained from surgical samples without adjuvant therapy. The clinical characteristics of all patients are listed in Additional file 5: Table S2. Additional 230 paraffin-embedded specimens were collected from endoscopic patients diagnosed with chronic non-atrophic gastritis (80 cases), intestinal metaplasia (50 cases), dysplasia (60 cases) and gastric cancers (40 cases). All specimens were provided by The First Affiliated Hospital of Nanchang University. The study protocol and exemption of informed consent were approved by the Ethics Committee of The First Affiliated Hospital of Nanchang University. Status of $H$. pylori infection for these clinical specimens was determined with a rapid urease test and Giemsa staining. Immunohistochemical staining was performed to examine expression profiles of YAP, E-cadherin and YAP/TAZ on these samples as described previously [29], which were evaluated and scored for intensity (scaled $0-3$ ) and frequency (scaled $0-4$ ) by two pathologists blinded to sample identity. For statistical analysis, expression levels of YAP and E-cadherin proteins were illustrated by an expression score in range of 0 to 12 using the formula intensity $\times$ frequency [30].

\section{Statistical analysis}

All the statistical analysis was performed using SPSS 20.0 software. Data were presented as mean \pm standard deviation (SD) of three independent experiments. Statistical significance of the in vitro studies for continuous variables were determined by one-way Analysis of variance (ANOVA) and Student's t-test. All comparison protein expression and clinicopathological parameters were performed with Kruskal-Wallis ( $>2$ groups) or Mann-Whitney tests (2 groups). Pearson correlation analysis was performed for the correlation between YAP and E-cadherin expression. $P$ value $\leq 0.5$ was considered significant (***, $P<0.001$, **, $P<0.01, *, P<0.05$ ).

\section{Results}

Expression of YAP was upregulated in human gastric cancer tissues and was correlated with tumour sizes and metastatic status

To determine the clinical relevance of YAP expression to the development of human gastric cancer, we assessed and compared expression patterns of YAP using immunohistochemistry in cancerous tissues versus their adjacent normal tissues via tumour resection collected from 199 gastric adenocarcinoma patients. YAP was distributed in both the cytoplasm and nucleus in the majority of gastric tumours (Fig. 1a). Compared with the adjacent noncancerous tissue, expression of YAP was significantly increased in gastric cancer tissues (Fig. 1b). Additionally, higher levels of cytoplasmic YAP were noted in the early tumour stages, whereas YAP was predominantly located in the nucleus at the advanced tumour stages (Fig. 1a, b). We also examined the association of YAP expression with pathologic severity of patients with gastric carcinoma. In addition, the transcriptional co-activator with PDZ-binding motif (TAZ), a paralog of YAP, was also overexpressed in gastric tumours compared with noncancerous tissues (Additional file 1: Figure S1A and B). While YAP and TAZ expression were not associated with gender, age of patients and location (Additional file 4: Table S1 and Additional file 5: Table S2), increased YAP and TAZ levels in the gastric tissues was positively correlated with invasion depth and lymph node metastasis (Fig. 1c, d; Additional file 1: Fig. S1C and D). These data suggested that elevated expression and nuclear translocation of YAP were associated with growth of tumour sizes and metastasis.

\section{Activation of YAP and suppression of E-cadherin} expression was positively correlated with progression of chronic non-atrophic gastritis to gastric cancer and $H$. pylori infection

Progression of chronic non-atrophic gastritis to intestinal metaplasia is a crucial step in the histopathological 


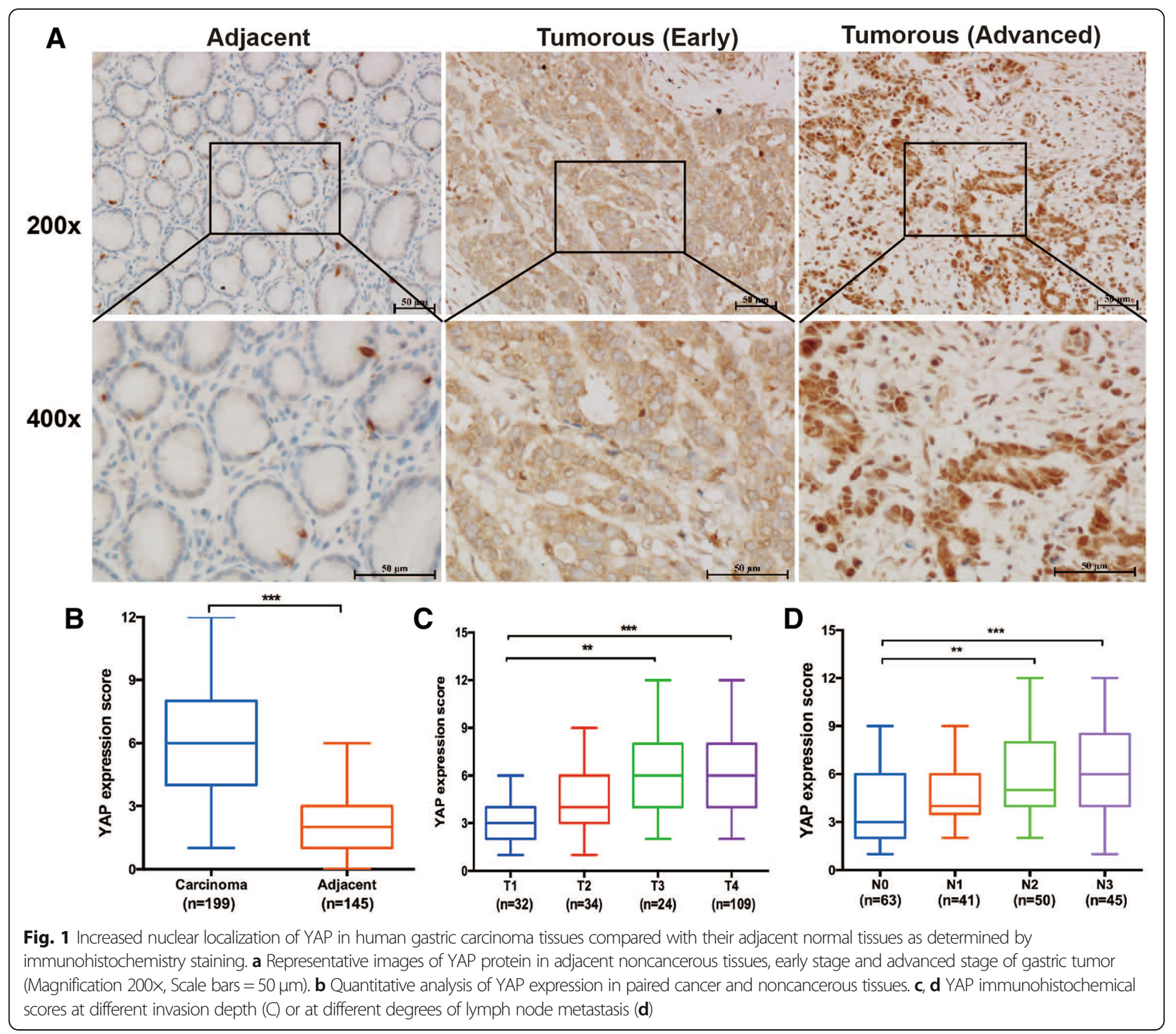

Correa cascade of gastric tumorigenesis [31]. Additionally, accumulating evidence suggests that activation of the YAP signalling pathway stimulates EMT, a key step for malignant transformation [32]. To explore the potential roles of the YAP signalling pathway and EMT in promoting the progression of gastric tumorigenesis in humans, we examined expression and cellular distribution of YAP and E-cadherin (an epithelial marker for EMT) in 230 human gastric tissues with CNAG, IM, Dys or GC using immunohistochemistry staining. YAP was present in both cytoplasm and nucleus (Fig. 2a), whereas E-cadherin staining was predominantly at the cell membrane (Fig. 2b). Data obtained from quantification of epithelial staining intensity and density indicated YAP levels were gradually increased during neoplastic progression (Fig. 2c), whereas there was a gradual decrease in the expression of E-cadherin (Fig. 2d). Pearson correlation analysis predicted that the levels of YAP expression were negatively correlated with the levels of E-cadherin (Fig. 2e).

Epidemiological data indicate that $H$. pylori is a major risk factor for the development of gastric cancer [33]. To characterize how $H$. pylori infection influenced expression of gastric YAP and E-cadherin, clinical samples were grouped into $H$. pylori ${ }^{+}$and $H$. pylori ${ }^{-}$subjects. We found that $H$. pylori $i^{+}$gastric tissues contained significantly higher levels of YAP (Fig. 2 f, $P<0.01$ ) and lower levels of E-cadherin (Fig. $2 \mathrm{~g}, P<0.01$ ) compared to H. pylori ${ }^{-}$tissues for CNAG but not for IM, Dys and GC (Fig. 2f, g). These results suggest that $H$. pylori infection elevated YAP expression in concert with reduction of E-cadherin in the early stage of the gastric tumorigenesis cascade, which could promote EMT and the possibility of eventual gastric cancer via induction of the YAP signalling pathway. 


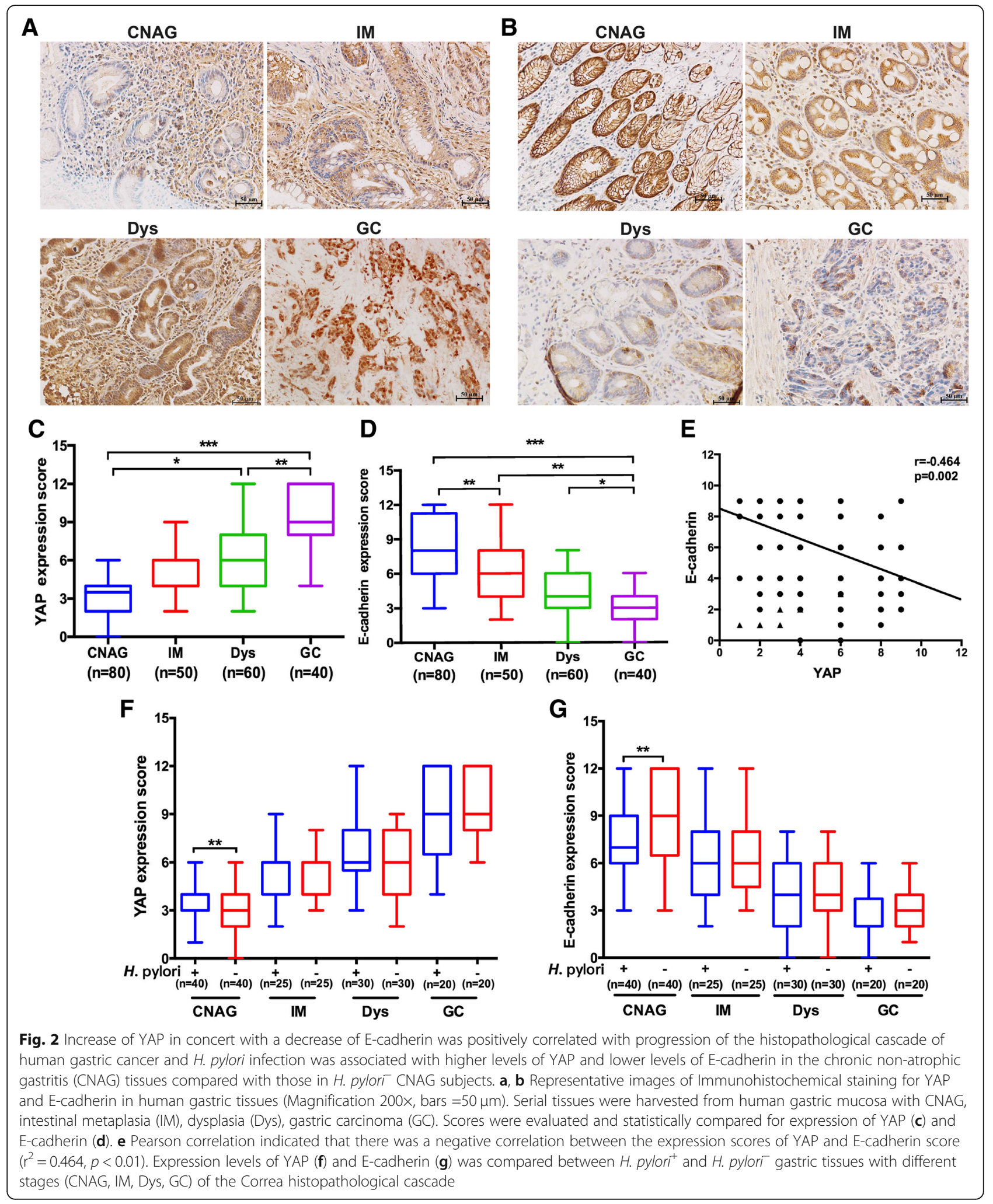




\section{$\mathrm{CagA}^{+} H$. pylori infection elevated YAP expression in gastric epithelial cells}

To dissect the possible mechanisms underlying $H$. pylor$i$-associated up-regulation of YAP in clinical subjects with chronic non-atrophic gastritis and characterize a role of CagA, a defined virulence effector of $H$. pylori-induced gastric tumorigenesis, in promoting the YAP pathway, we infected Gastric cell lines AGS with $c a g A^{+}$ and $\operatorname{cag} A^{-} H$. pylori strains. To avoid confusion of CagA status of $H$. pylori strains, $\mathrm{CagA}^{+}$and $\mathrm{CagA}^{-}$are used to represent a given $H$. pylori strain with or without functional CagA throughout the text, respectively. Functional CagA means that this protein can be delivered into and induce cytopathic effects on the host cells. Gastric epithelial AGS cells were co-cultured with $\mathrm{CagA}^{+} H$. pylori strains PMSS1 and 7.13 as well as $\mathrm{CagA}^{-} H$. pylori strain SS1 at different MOIs (50, 100 and 200) for 6 or $24 \mathrm{~h}$. $H$. pylori SS1, a mouse-adapted strain of PMSS, produces CagA in bacterial cells, but it can't be delivered into the host cells due to mutations in $\operatorname{cag} Y$ [34]. Infection with strains PMSS1 and 7.13 significantly increased levels of total YAP in AGS cells with a trend in a MOI-dependent manner at $6 \mathrm{~h}$ post infection (HPI) (Fig. 3a, b). However, infection with $\mathrm{CagA}^{+}$strains PMSS1 and 7.13 did not affect the ratio of Ser127-phosphorylated YAP to total YAP at 6 HPI (Additional file 2: Fig. S2A and B); this $H$. pylori-induced elevation of YAP was diminished at $24 \mathrm{HPI}$ (Additional file 2: Fig. S2C). In addition, there was MOI-dependent transcriptional increase of $Y A P$ and its downstream target genes CTGF and CYR61, most significantly at MOI of 200, in the AGS cells infected with $H$. pylori PMSS1 or 7.13 compared with uninfected controls at $6 \mathrm{HPI}$ (Fig. 3c). In contrast, $\mathrm{CagA}^{-} H$. pylori SS1 infection did not significantly enhance YAP levels compared to that in controls at $6 \mathrm{HPI}$ (Additional file 2: Fig. S2D).

\section{Construction and characterization of PMSS1 isogenic $\triangle$ cagA mutants}

To directly characterize the effect of CagA on the YAP signalling pathway, we constructed isogenic PMSS1 $\triangle$ cagA mutants. The entire $\operatorname{cag} A$ region containing variable cagA copies in the PMSS1 population was replaced with a chloramphenicol resistance cat cassette (Fig. 4a, Additional file 3: Fig. S3A and B) [35]. Isogenic PMSS1 $\triangle$ cagA mutants were generated by introducing the recombinant plasmids (Additional file 3: Fig. S3C) into recipient PMSS1 cells using electrotransformation or natural transformation, followed by selection on blood agar plates with chloramphenicol. $\mathrm{Cm}^{\mathrm{R}}$ PMSS1 clones were determined for authenticity of deletion and the cat orientation in the PMSS1 genome by PCR assays using specific primers detailed in the Materials and Methods and Additional file 5: Table S2. A PCR amplicon with two specific primer pairs F1/R1 and F2/R2, which targeted the 5 '-end and 3 '-end regions of cagA respectively, was produced from chromosomal DNA templates only from PMSS1, but not from $\mathrm{Cm}^{\mathrm{R}}$ PMSS1 clones, confirming the complete deletion of $\operatorname{cagA}$ (Fig. 4b). The transcriptional orientation of cat, either opposite or correspondingly to the transcriptional orientation of $\operatorname{cag} A$ in the mutated $H$. pylori genome, was determined using two primer pairs Fup/54 and Fup/55, respectively (Fig. 4c). Four mutants, designated 54E, 55E, $54 \mathrm{~N}$ and $55 \mathrm{~N}$ were selected for further characterization. 54 and 55 represented that the transcriptional orientation of cat, opposite to $\operatorname{cag} A$ (54) and along with $\operatorname{cagA}$ (55) respectively, whereas $\mathrm{E}$ and

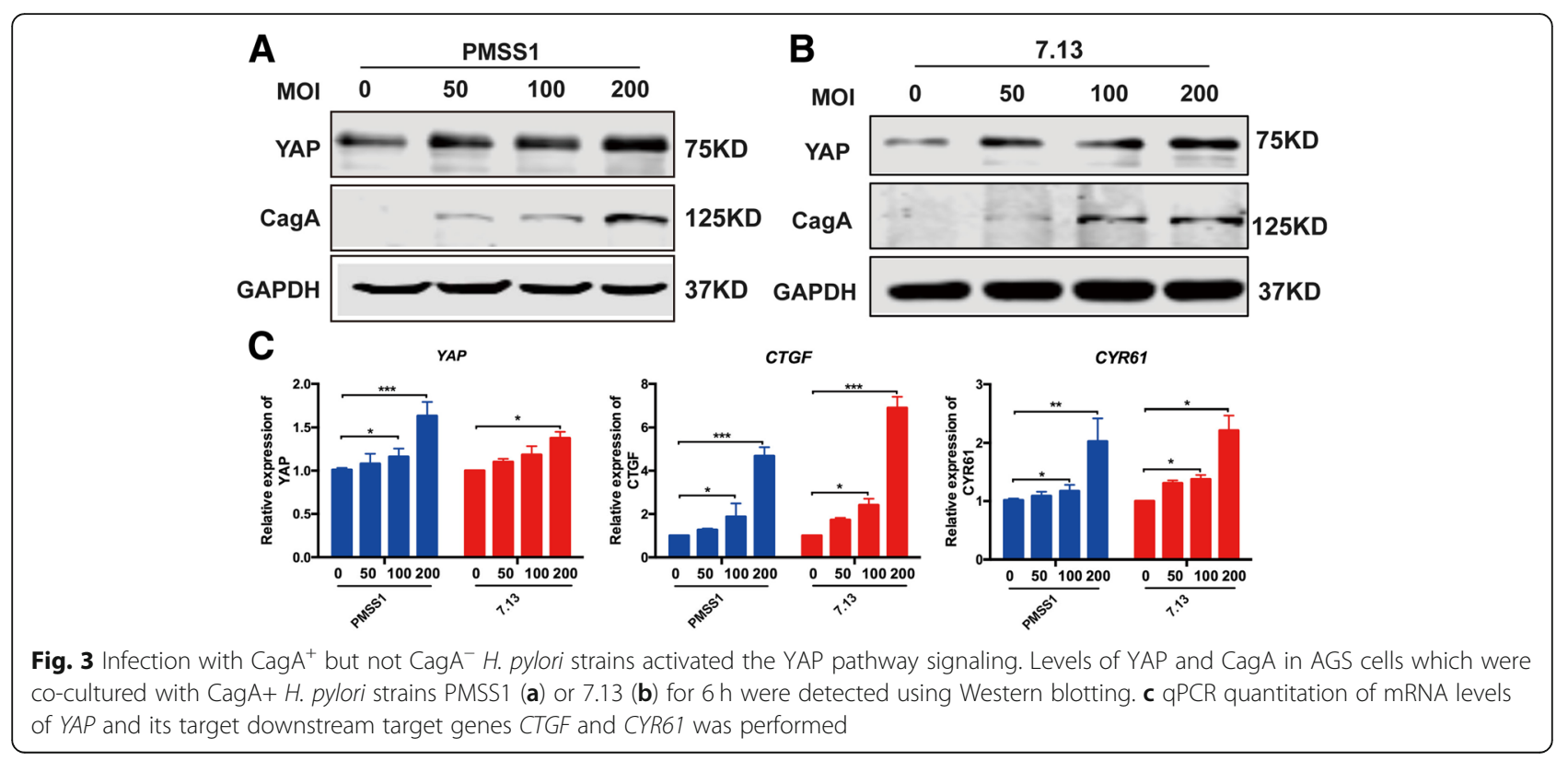




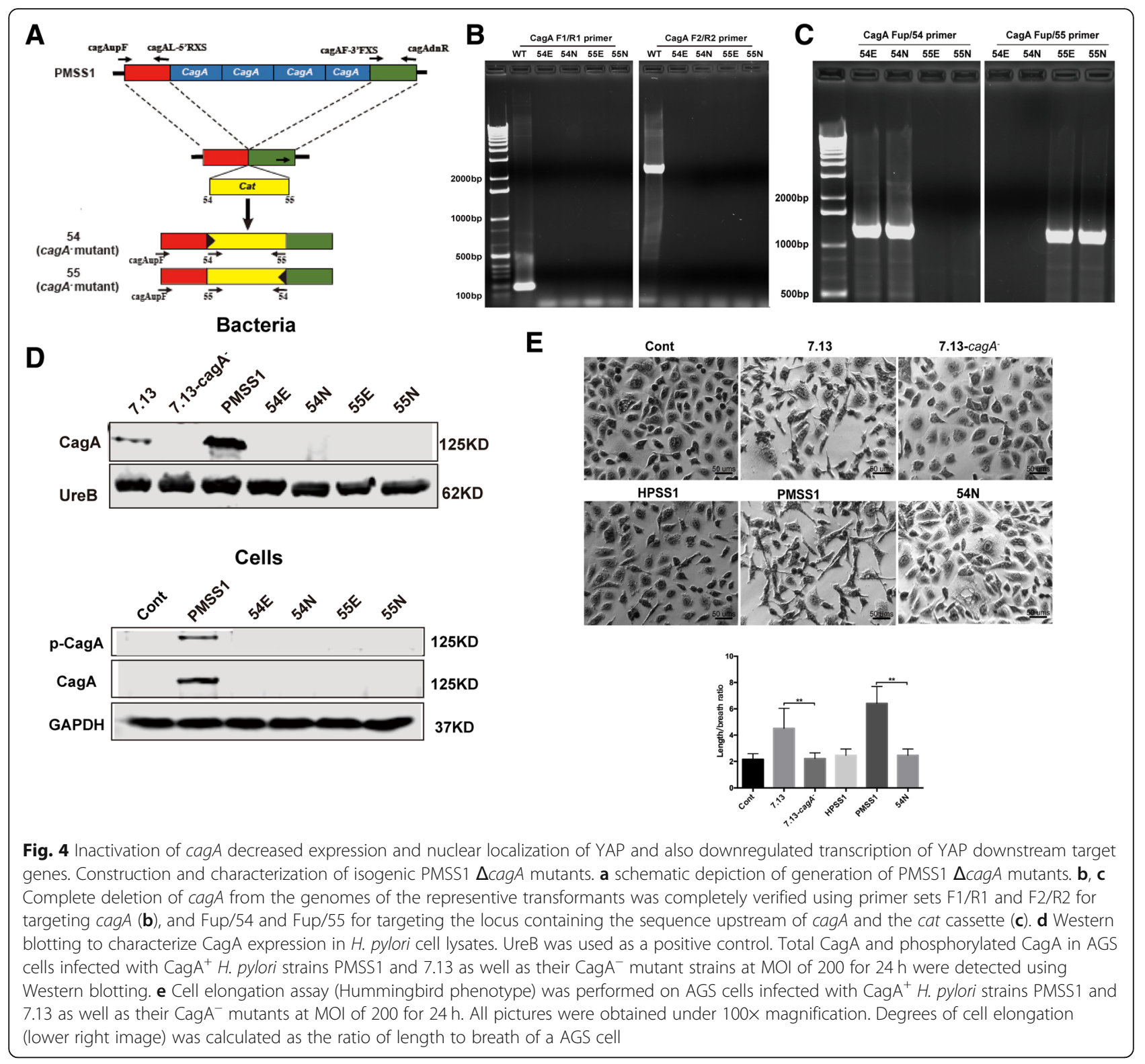

$\mathrm{N}$ represented the mutants obtained by electrotransformation $(\mathrm{E})$ or natural transformation $(\mathrm{N})$ (see their identity in Additional file 6: Table S3).

The loss of CagA in the PMSS1 $\triangle$ cagA mutants was further characterized by examining CagA expression and the ability of these mutants to translocate CagA into AGS cells and cause a cytopathic "hummingbird" phenotype. CagA was present in cell lysates from PMSS1 and 7.13, but was absent from all 4 PMSS1 $\triangle$ cagA mutants and the 7.13 cagA- mutant. In addition, CagA translocation and phosphorylation was detected in the AGS cells co-cultured with parental strains PMSS1 and 7.13 but not with their respective mutants (Fig. 4d and Additional file 3: Fig. S3D). After delivery into gastric epithelial cells, CagA induced the "hummingbird" phenotype [13].
Using this assay, AGS cells infected with $\mathrm{CagA}^{+} H$. pylori strains PMSS1 and 7.13 exhibited significant cell elongation compared to uninfected controls, whereas the morphology of cells infected with $\mathrm{CagA}^{-} H$. pylori mutants, 7.13 cagA- or $54 \mathrm{~N}$, was not significantly different from those of the uninfected controls (Fig. 4e). In addition, there was no "hummingbird" phenotype on AGS cell co-cultured with CagA- H. pylori SS1. These results collectively demonstrated that PMSS1 $\triangle$ cagA mutants lost functional CagA.

\section{H. pylori CagA promoted the YAP pathway signalling}

To investigate the role of CagA in the regulation of the YAP signalling pathway, AGS cells were co-cultured with $\mathrm{CagA}^{+}$H. pylori strains 7.13 or PMSS1, and their 
corresponding $\mathrm{CagA}^{-}$mutants. Levels of YAP mRNA and protein in the cells infected with the $\mathrm{CagA}^{-}$strains were comparable to those in the uninfected control cells, whereas infection with PMSS1 and 7.13 significantly increased expression of YAP mRNA and protein when compared with the uninfected controls (Fig. 5a, b and f). Similar effects on YAP expression were obtained from another human gastric cancer cell line MKN-45 co-cultured with $\mathrm{CagA}^{+}$and $\mathrm{CagA}^{-}$ H. pylori strains (Fig. 5c, d). To further characterize whether elevated YAP enhances its function as a transcription activator, immunofluorescence staining, and $\mathrm{qPCR}$ were performed for determining the nuclear localization of YAP and expression of its downstream target genes respectively. Treatment with PMSS1 or 7.13 significantly induced the nuclear translocation of YAP, while infection with $H$. pylori strains lacking CagA resulted in cytoplasmic retention and inactivation of YAP as occurred within the uninfected control cells (Fig. 5e). In addition, levels of CTGF and CYR61 mRNA, whose transcription is controlled by the activation of YAP, were significantly elevated in the AGS cells infected with PMSS1 and 7.13 but not with their $\mathrm{CagA}^{-}$mutants compared to the uninfected controls (Fig. 5f). Furthermore, expression of the recombinant CagA protein vectored by plasmid name (KX673185) in AGS cells significantly elevated protein levels of YAP compared with its empty vector control (Fig. 5g). These data demonstrated that H. pylori CagA plays a crucial role in promoting activation of the YAP pathway signalling in this in vitro model.

\section{$\mathrm{CagA}^{+} H$. pylori infection enhanced EMT through the activation of the YAP pathway}

Given that the levels of YAP were inversely correlated with the levels of E-cadherin noted in the H. pylori ${ }^{+}$ CNAG tissues vs the H. pylori $i^{-}$CNAG tissues and also during the progression of the Correa cascade of human gastric tumorigenesis (Fig. 2c, g), we investigated whether CagA-dependent YAP induction could promote EMT in AGS cells. To evaluate the relationship between YAP expression and EMT, we introduced a plasmid containing the YAP cDNA into AGS cells. Transient overexpression of YAP decreased the level of E-cadherin, an epithelial marker of EMT, indicating that activation of YAP facilitates EMT (Fig. 6a). Treatment with wild-type H. pylori strains (7.13 or PMSS1) led to the reduction of E-cadherin expression compared with the uninfected controls as visualized by immunofluorescence, whereas E-cadherin expression were partially restored in the AGS cells infected with $\mathrm{CagA}^{-}$H. pylori mutants (Fig. 6b).

To further verify the role of YAP in promoting EMT, we treated AGS cells with verteporfin, a YAP inhibitor through disrupting YAP interaction with TEADs and promoting trypsin cleavage of YAP [36]. YAP expression was decreased by treatment with verteporfin in a concentration-dependent manner (Additional file 3: Fig. S3E). Treatment of verteporfin attenuated partially augmented E-cadherin expression (Fig. 6c) and $H$. pylori-induced mRNA levels of CTGF and CYR61 (Fig. 6d) and in CagA ${ }^{+}$H. pylori-infected AGS cells. In contrast, the levels of Slug (a repressor of E-cadherin expression) and N-cadherin (a mesenchymal marker of EMT) were decreased in the cells treated with both $H$. pylori and verteporfin compared with the cells treated with $H$. pylori alone (Fig. 6e). Furthermore, knockdown of YAP by YAP siRNA in vitro upregulated the epithelial marker E-cadherin which was inhibited by $H$. pylori CagA, and downregulated the mesenchymal marker $\mathrm{N}$-cadherin which was induced by $H$. pylori CagA (Fig. 6f). These findings indicate that activation of YAP played an important role in H. pylori infection-induced EMT.

Wound healing assays and Boyden chamber assays were also performed to assess the effect of $H$. pylori CagA on the migration and motility of gastric cancer cells. We found that infection with $H$. pylori strains PMSS1 or 7.13 significantly increased the migration of AGS cells, whereas infection with $\mathrm{CagA}^{-}$mutants of these strains inhibited this migratory phenotype (Fig.7a, b). In addition, treatment with a YAP inhibitor verteporfin in combination with $H$. pylori infection significantly suppressed $\mathrm{CagA}^{+} \mathrm{H}$. pylori-induced gastric cells invasion (Fig. 7c) and cells migration (Fig. 7d, e). Taken together, these findings suggested that activation of YAP promotes H. pylori CagA -induced cell invasion and migration.

\section{Discussion}

Epidemiological data indicate that the presence of functional CagA is associated with the higher risk for the development of gastric cancer [37]. It has also been documented in vitro and in vivo that CagA can trigger various pro-oncogenic signaling such as $\beta$-catenin, PI3K/Akt, Erk signaling pathways, thereby potentiating the ability of $H$. pylori to induce gastric carcinogenesis [38]. YAP as a key effector of the Hippo signaling pathway is involved in appropriate cellular functionality such as cell proliferation, differentiation, migration and gastric epithelial cells homeostasis. In this study, we showed that there was more YAP expression and stronger the nuclear translocation of YAP in H. pylori + CNAG compared with H. pylori CNAG, whereas opposite was true for E-cadherin expression. Subsequently, we demonstrated that CagA functioned as an inducer of the pro-oncogenic YAP signaling pathway in AGS cells, a new mechanism possibly underlying the $H$. pylori-associated elevation of YAP in concert with the decrease of E-cadherin expression noted in human subjects, to promote gastric carcinogenesis. Infection of $\mathrm{CagA}^{+} H$. pylori strains PMSS1 and 7.13 as well as recombinant CagA expression led to upregulation of YAP expression, increased nuclear localization of YAP, enhanced expression of downstream genes CTGF and CYR61, decreased expression of E-cadherin, eventually induced invasion and migration of AGS cells, while these cytopathic 


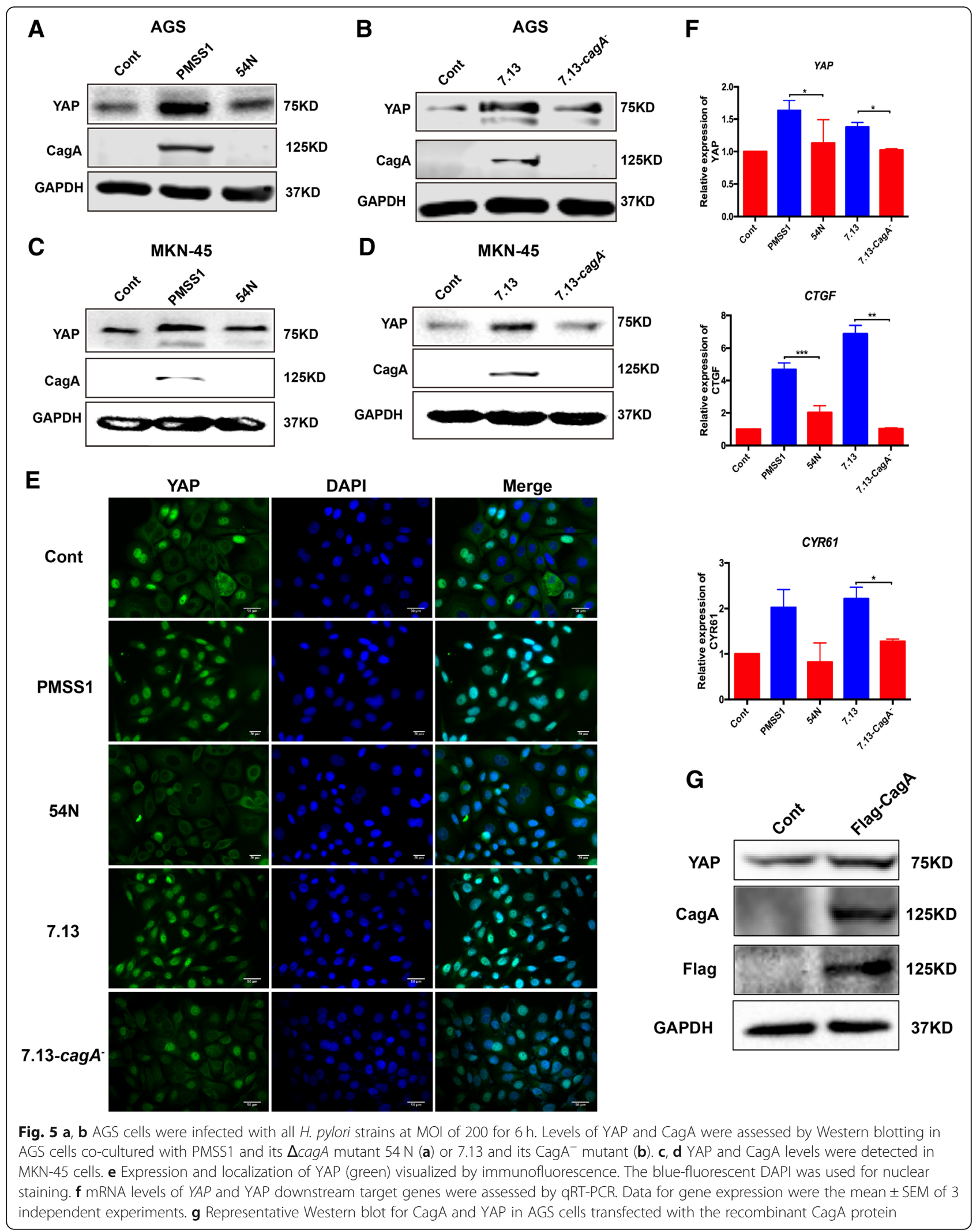


A

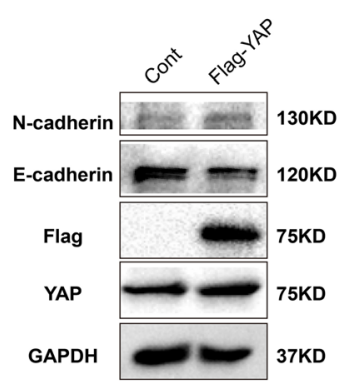

C

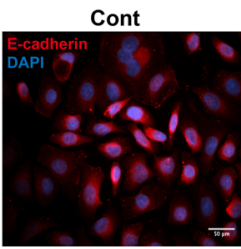

VP

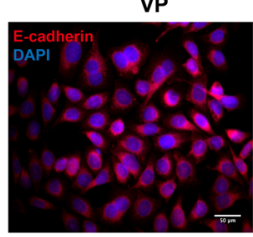

E

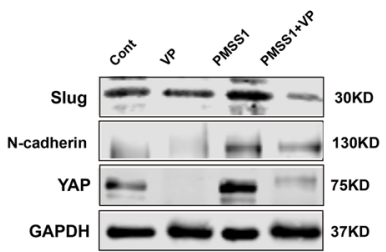

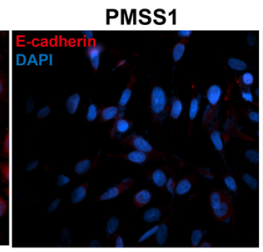

PMSS1+VP

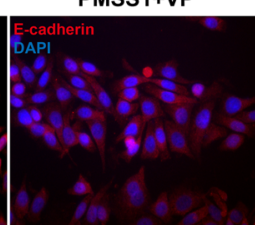

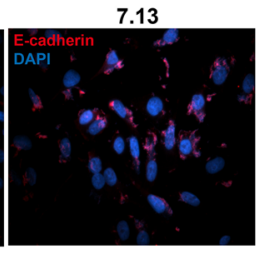

7.13+VP

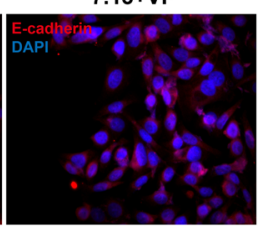

B
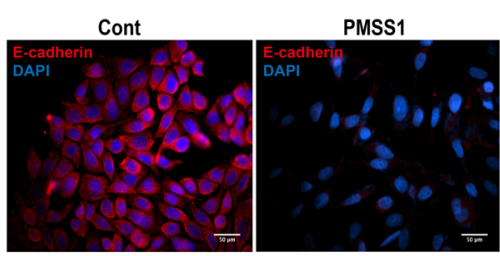

$54 N$

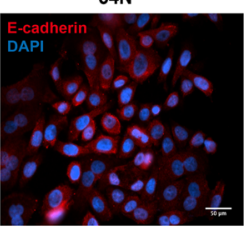

D
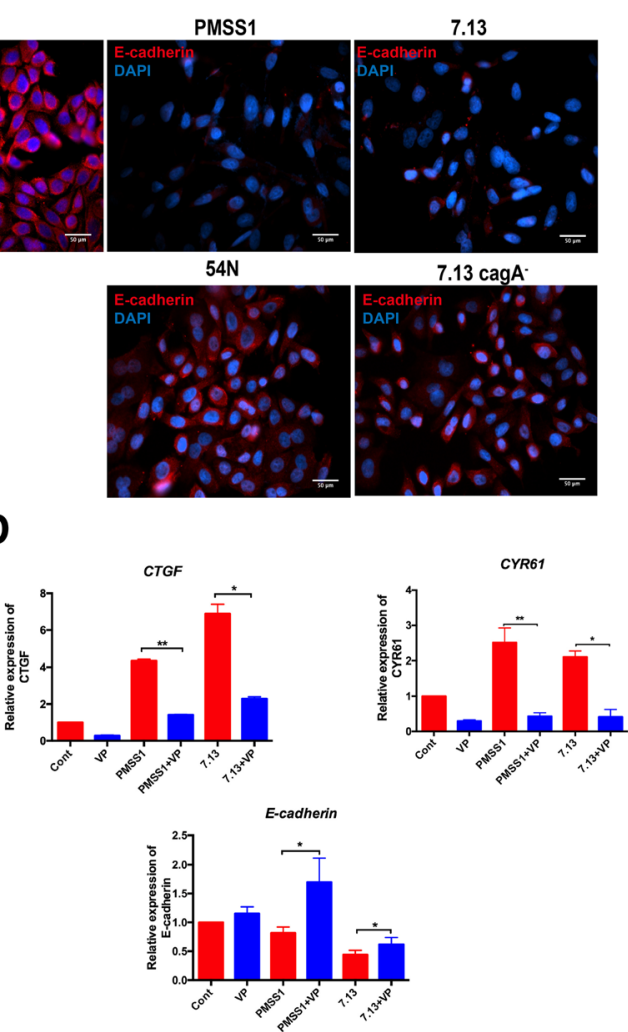

$7.13 \mathrm{cagA}$.

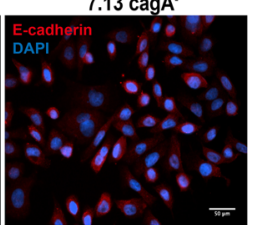

F

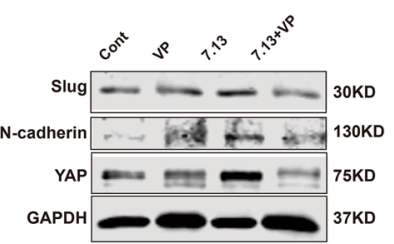

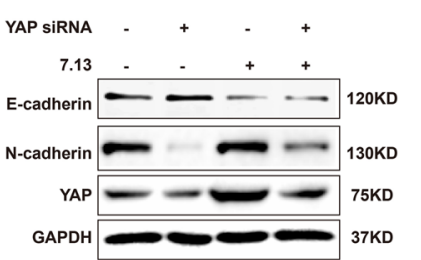

Fig. 6 a Expression of E-cadherin (an epithelial marker) and N-cadherin (a mesenchymal marker) in AGS cells transfected with YAP cDNA plasmid. b After AGS cells were infected with H. pylori strains 7.13 or PMSS1 and their cagA $A^{-}$mutants, Expression of E-cadherin (red) were visualized using Immunofluorescence. c Immunofluorescence was performed for E-cadherin levels. d mRNA levels of YAP downstream genes CTGF, CYR61 and E-cadherin (an epithelial marker) in AGS cells infected with $\mathrm{H}$. pylori strains alone or in combination with VP treatment. e Expression of YAP and mesenchymal markers Slug and N-cadherin in AGS cells treated with $\mathrm{CagA}^{+}$H. pylori PMSS1 or 7.13 alone or in combination with VP. $\mathbf{f}$ Expression of YAP, epithelial markers E-cadherin and mesenchymal markers $\mathrm{N}$-cadherin in AGS cells treated with CagA ${ }^{+}$. pylori PMSS1 or 7.13 alone or in combination with YAP siRNA. Data for gene expression are mean \pm SEM of 3 independent experiments

effects were suppressed by inactivation of CagA from these H. pylori strains or by treatment with verteporfin, a YAP inhibitor or by YAP siRNA. Based on these findings, we propose a working model to illustrate the mechanism underlying the role of CagA in promoting gastric tumorigenesis (Fig. 8). In this model, CagA ${ }^{+} H$. pylori infection further elevates activation of the YAP pathway signaling that enhances EMT (a hallmark of tumorigenic transformation) via skewed expression of mesenchymal factors such as $\mathrm{N}$-cadherin and Slug, thereby promoting cell migration and gastric tumorigenesis.

A previous study showed that there were elevated mRNA levels of YAP and its downstream targets CTGF, CYR61 and CDX2 in the gastric tissues of C57BL/6 mice treated with a carcinogenic agent MNNG in combination with $\mathrm{CagA}^{-} H$. pylori SS1 infection when compared with uninfected controls [26]. This finding appears to be in disagreement with our results that $H$. pylori SS1 and $\mathrm{CagA}^{-}$mutants of $H$. pylori strains PMSS1 and 7.13 did not enhance expression of YAP and its downstream genes CTGF and CYR61 in AGS cells. At least two factors could contribute to this discrepancy. First, experimental systems used in these two studies are fundamentally different. The AGS cell line used in our study were originally derived from a gastric cancer tissue [39]. The direct interaction between AGS cells and $H$. pylori allowed analysis under a more defined condition. In contrast, the responses of C57BL/6 mice to $H$. pylori 


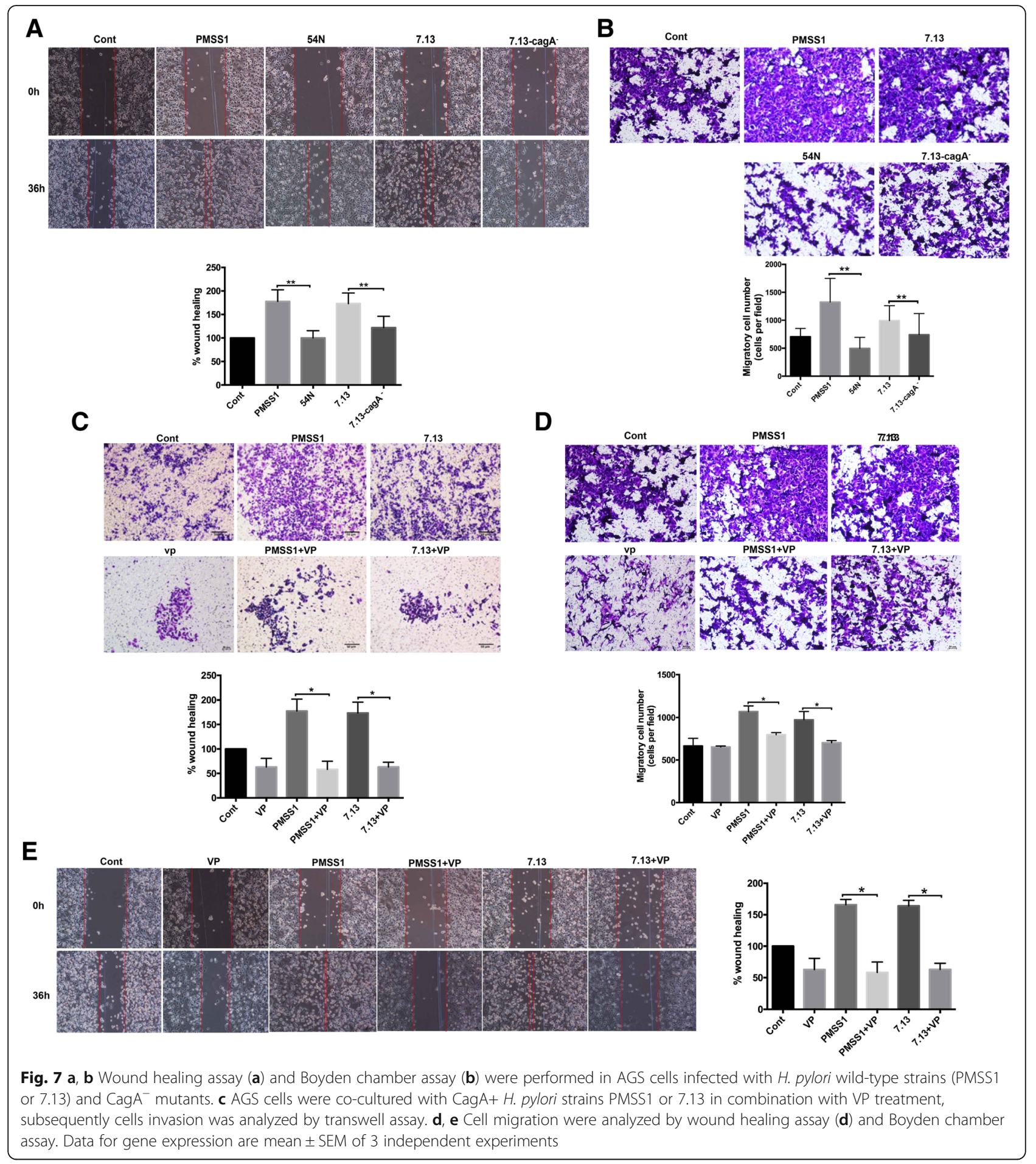

infection are modulated by a more complicated environment full of cytokines and immune cells involved in pro-inflammatory and anti-inflammatory pathways. Second, in the study of Jiao et al. [26], it is unclear how treatment with MNNG alone or $H$. pylori alone influenced Yap expression in C57BL/6 mice, because these groups were not described in their study.
EMT is frequently activated in cancer invasion and metastasis, and it also contributes to the initiation of gastric adenocarcinoma. During this process, activated EMT reduces gastric epithelial features and confers mesenchymal characteristics [40]. Our results showed that infection with $\mathrm{CagA}^{+} H$. pylori strain PMSS1 or 7.13 led to downregulation of the epithelial marker 


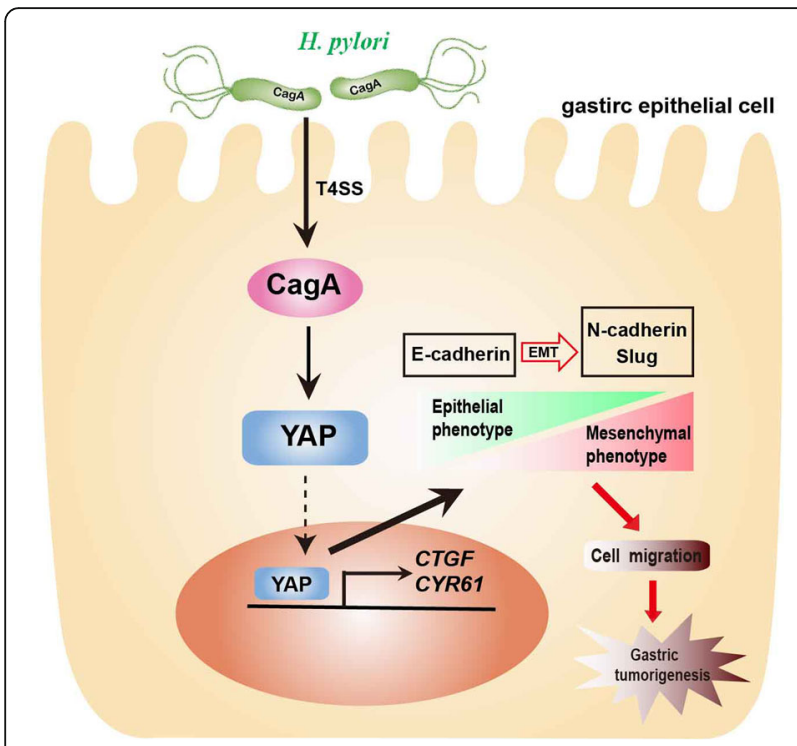

Fig. 8 The proposed mechanism of H. pylori CagA-mediated promotion of gastric tumorigenesis. CagA $\mathrm{A}^{+} \mathrm{H}$. pylori delivers $\mathrm{CagA}$ into gastric epithelial cells via T4SS where CagA induces oncogene YAP expression, increases nuclear translocation of YAP that elevates downstream gene expression. The H. pylori CagA-mediated activation of YAP then leads to enhanced EMT program, thereby further promoting gastric tumorigenesis

E-cadherin expression in concert with increased expression of a mesenchymal marker $\mathrm{N}$-cadherin, further promoting invasion and migration of gastric epithelial cancer cell lines AGS. Importantly, CagA deficiency in $H$. pylori strains PMSS1 and 7.13 or treatment of verteporfin suppressed YAP expression and partially restored E-cadherin expression and inhibited cell migration. The partial restoration of E-cadherin in the $\mathrm{CagA}^{-} H$. pylori-infected AGS cells suggested that additional $H$. pylori factors could also contribute to EMT. Our results are also consistent with a previous study reporting that induction of EMT on AGS cells by $H$. pylori strain 60190 was a CagA-dependent process [25]. Mechanistically, it has been reported that the EPIYA motif of CagA can bind to GSK-3, resulting in depletion of GSK-3 and abnormal expression of various cancer-associated genes including AMPK, $\beta$-catenin. Intriguingly, recent study has indicated that YAP enhances the transcriptional activity of $\beta$-catenin via GSK-3 activity in glioma progression [41]. Also, the cellular energy sensor AMPK exerts an inhibitory role on the YAP activity via promotion of its phosphorylation [42]. Therefore, these studies suggest that enhanced YAP pathway due to $H$. pylori infection is probably associated with GSK3/ $\beta$-catenin or AMPK pathway regulated by CagA. Of note, it has been reported that the Hippo signaling pathway suppresses tumorigenesis by phosphorylation of YAP on Ser127, which can restrict YAP to the cytoplasm where phosphorylated YAP undergoes sequestration via binding to 14-3-3 protein. However, in our study $\mathrm{CagA}^{+} H$. pylori infection elevated the levels of total YAP, but did not alter the ratio of phospho (Ser 127)-YAP to the total YAP. It is likely that the increase of YAP nuclear translocation and upregulation of YAP target genes in the CagA ${ }^{+}$H. pylori-infected AGS cells result from the elevation of unphosphorylated YAP.

It has been proposed that gastric cancer is gradually developed over many years through a multistep histopathological cascade [4]. In this study, we showed that gastric YAP expression also increased, which was correlated with the decrease of E-cadherin, from the non-atrophic gastritis, dysplasia to gastric cancer, further suggesting that the YAP signaling pathway plays a pivotal role in promoting gastric tumorigenesis. More importantly, higher YAP levels and lower E-cadherin levels were noted in the chronic gastritis tissues from $H$. pylori-positive patients compared to those from $H$. pylori-negative patients, suggesting that activation of the YAP signalling pathway at the stage of CNAG is one of the major molecular mechanisms $H$. pylori utilizes to promote the cascade of gastric carcinogenesis. This hypothesis is supported by the results that the promotion effect of $\mathrm{CagA}^{+} H$. pylori on YAP expression was diminished at the stages of IM/Dys/GC and also is consistent with our data that enhancement of YAP expression in the $\mathrm{CagA}^{+} H$. pylori-infected AGS cells occurred at 6 HPI and diminished at 24 HPI. Future investigations into the correlation between $H$. pylori $\mathrm{CagA}^{+}$ status and YAP expression in clinical samples will further strengthen the role of CagA in increasing a GC risk via activation of the YAP signaling in the early stage of the development of gastric cancer.

In summary, we have demonstrated that H. pylori CagA promotes gastric tumorigenesis via activation of the YAP signalling pathway in AGS cells during the initiation of $H$. pylori infection. These in vitro results are further linked to the clinical observation that $H$. pylori infection elevated activation of the YAP expression in concert with the suppression of E-cadherin in the chronic gastritis tissues of $H$. pylori + patients compared with those of $H$. pylori- patients. These data have highlighted an important role of YAP in CagA virulence potential in particular and gastric tumorigenesis in general. Thus, the findings from this study may have provided a mechanistic insight into why $\operatorname{cag} A^{+} H$. pylori infection is associated with a higher risk for the development of gastric cancer. In addition, further investigations into the correlation among $H$. pylori cagA status, YAP expression and chronic gastritis could potentially develop novel strategies for eradicating $H$. pylori and prevent the development of gastric cancer.

\section{Conclusions}

Our data reveal that $H$. pylori infection activates the YAP signalling pathway to promote EMT in gastric carcinogenesis via CagA, a defined $H$. pylori virulence 
factor. These findings not only highlight a new mechanistic insight into the role of CagA in promoting $H$. pylori-induced gastric carcinogenesis, but also provide a novel molecular target for developing effective strategies to eradicate $H$. pylori and prevent the development of gastric cancer.

\section{Additional files}

\section{Additional file 1: Figure S1. (A) Representative images of} Immunohistochemistry staining of TAZ in human gastric carcinoma tissues. (B) Quantitative analysis of YAP expression in paired cancer and noncancerous tissues. (C, D) YAP immunohistochemical scores at different invasion depth (C) or at different degrees of lymph node metastasis (D). (JPG $198 \mathrm{~kb}$ )

Additional file 2: Figure S2. (A, B) Phosphorylation of YAP was detected using western blotting in AGS cells infected with H. pylori 7.13 (A) or PMSS1 (B) strain, respectively for $6 \mathrm{~h}$. (C) Western blotting was performed for YAP expression in AGS cells cocultured with CagA $A^{+} H$. pylori PMSS1 strains for $24 \mathrm{~h}$. (D) YAP and CagA were assessed in AGS cells cocultured with CagA $\mathrm{A}^{-}$H. pylori SS1 strain, at 6 h' time point. (JPG $204 \mathrm{~kb}$ )

Additional file 3: Figure S3. Generation and characterization of PMSS1 $\triangle$ cagA mutants. (A) Overlapping PCR amplicon consisting of the upstream and downstream regions of the PMSS1 cagA gene. (B) A 0.7-kb cat (chloramphenicol acetyltransferase) cassette digested with Hincll. (C) Smal-digested recombinant plasmid containing the upstream and downstream regions of the PMSS1 cagA gene. (D) CagA and phospho-CagA were assessed using Western blotting in AGS cells infected with PMSS1 and its isogenic $\triangle$ cagA mutants at different MOI for $6 \mathrm{~h}$. (E) Effect of different concentrations of verteporfin (NP) on YAP expression. (JPG $1527 \mathrm{~kb}$ )

Additional file 4: Table S1. Correlation of YAP expression and clinic pathological status of the patient with GC. (DOCX $14 \mathrm{~kb}$ )

Additional file 5: Table S2. Correlation of TAZ expression and clinic pathological status of the patient with GC. (DOCX $14 \mathrm{~kb}$ )

Additional file 6: Table S3. The primers used in this study. (DOCX 13 kb)

\section{Abbreviations}

cagA: Cytotoxin-associated gene A; CNAG: Chronic non-atrophic gastritis; CTGF: Connective tissue growth factor; CYR61: Cysteine-rich angiogenic inducer 61; DAPI: 6-diamidino-2-phenylindole; Dys: Dysplasia; EMT: Epithelial-mesenchymal transition; FITC: Fluorescein isothiocyanate; GC: Gastric cancer; H. pylori: Helicobacter pylori; IM: Intestinal metaplasia; MNNG: Administration of 1-methyl-3-nitro-1-nitrosoguanidine; MOI: Multiplicity of infection; YAP: Yes-Associated Protein

\section{Acknowledgements}

The funding body had no role in study design, data collection and analysis, decision to publish, or preparation of the manuscript.

\section{Funding}

This work was supported by China Scholarship Council, The Graduate Innovation Fund of Jiangxi Province (YC2016-B023), National Natural Science Foundation of China (81270479, 81470832, and 81670507), Grants from the National Science and Technology Major Projects for "Major New Drugs Innovation and Development" of China (2011ZX09302-007-03).

\section{Availability of data and materials}

The datasets supporting the conclusions of this article are included within the article and additional files.

\section{Authors' contributions}

NSL, ZJL, ZMG, NHL conceived and designed the study. NSL and YF performed in vitro experiments and analysed the data in AGS cells; NSL, YBOY, DQH collected human specimens and analysed immunohistochemical data. YH, CH, CX, SCA, YZ provided assistance with analyses of the data and technical issues. NSL, YH, ZMG interpreted the data and drafted the manuscript. NHL, ZMG supervised and oversaw the study. All the authors critically revised the manuscript and provided intellectual content. All authors read and approved the final manuscript.

\section{Ethics approval and consent to participate}

This study was approved by the ethics committee of The First Affiliated Hospital of Nanchang University (2016034), and written informed consent was obtained from all patients.

\section{Consent for publication}

Not applicable.

\section{Competing interests}

The authors declare that they have no competing interests.

\section{Publisher's Note}

Springer Nature remains neutral with regard to jurisdictional claims in published maps and institutional affiliations.

\section{Author details}

'Department of Gastroenterology, The First Affiliated Hospital of Nanchang University, 17 Yong Waizheng Street, Donghu District, Nanchang 330006, Jiangxi Province, China. ${ }^{2}$ Division of Comparative Medicine, Massachusetts Institute of Technology, 77 Massachusetts Avenue, Cambridge, MA 02139, USA. ${ }^{3}$ Department of Biochemistry, Boston University School of Medicine, 72 East Concord Street, Boston, MA 02118, USA.

Received: 30 July 2018 Accepted: 14 November 2018 Published online: 22 November 2018

\section{References}

1. Malaty HM, El-Kasabany A, Graham DY, Miller CC, Reddy SG, Srinivasan SR, Yamaoka Y, Berenson GS. Age at acquisition of helicobacter pylori infection: a follow-up study from infancy to adulthood. Lancet. 2002;359(9310):931-5.

2. Weyermann M, Rothenbacher $\mathrm{D}$, Brenner H. Acquisition of Helicobacter pylori infection in early childhood: independent contributions of infected mothers, fathers, and siblings. Am J Gastroenterol. 2009;104(1):182-9.

3. Wu MS, Chow LP, Lin JT, Chiou SH. Proteomic identification of biomarkers related to helicobacter pylori-associated gastroduodenal disease: challenges and opportunities. J Gastroenterol Hepatol. 2008;23(11):1657-61.

4. Fox JG, Wang TC. Inflammation, atrophy, and gastric cancer. J Clin Invest. 2007:117(1):60-9.

5. Cover TL, Peek RM Jr. Diet, microbial virulence, and helicobacter pyloriinduced gastric cancer. Gut Microbes. 2013;4(6):482-93.

6. Fox JG, Wang TC. Dietary factors modulate helicobacter-associated gastric cancer in rodent models. Toxicol Pathol. 2014;42(1):162-81.

7. Parsonnet J, Friedman GD, Orentreich N, Vogelman H. Risk for gastric cancer in people with CagA positive or CagA negative helicobacter pylori infection. Gut. 1997:40(3):297-301.

8. Ohnishi N, Yuasa H, Tanaka S, Sawa H, Miura M, Matsui A, Higashi H, Musashi M, Iwabuchi K, Suzuki M, et al. Transgenic expression of helicobacter pylori CagA induces gastrointestinal and hematopoietic neoplasms in mouse. Proc Natl Acad Sci U S A. 2008:105(3):1003-8.

9. Chaturvedi R, Asim M, Romero-Gallo J, Barry DP, Hoge $\mathrm{S}$, de Sablet T, Delgado AG, Wroblewski LE, Piazuelo MB, Yan F, et al. Spermine oxidase mediates the gastric cancer risk associated with Helicobacter pylori CagA. Gastroenterology. 2011;141(5):1696-708 e1-2.

10. Odenbreit S, Puls J, Sedlmaier B, Gerland E, Fischer W, Haas R. Translocation of helicobacter pylori CagA into gastric epithelial cells by type IV secretion. Science. 2000:287(5457):1497-500.

11. Hatakeyama M. Helicobacter pylori CagA and gastric cancer: a paradigm for hit-and-run carcinogenesis. Cell Host Microbe. 2014;15(3):306-16.

12. Higashi $H$, Tsutsumi R, Muto S, Sugiyama T, Azuma T, Asaka M, Hatakeyama M. SHP-2 tyrosine phosphatase as an intracellular target of helicobacter pylori CagA protein. Science. 2002;295(5555):683-6.

13. Bourzac KM, Botham CM, Guillemin K. Helicobacter pylori CagA induces AGS cell elongation through a cell retraction defect that is independent of Cdc42, Rac1, and Arp2/3. Infect Immun. 2007;75(3):1203-13.

14. Yu FX, Zhao B, Guan KL. Hippo pathway in organ size control, tissue homeostasis, and Cancer. Cell. 2015;163(4):811-28.

15. Piccolo S, Dupont S, Cordenonsi M. The biology of YAP/TAZ: hippo signaling and beyond. Physiol Rev. 2014;94(4):1287-312. 
16. Moroishi T, Hansen CG, Guan KL. The emerging roles of YAP and TAZ in cancer. Nat Rev Cancer. 2015;15(2):73-9.

17. Li N, Xie C, Lu N. Crosstalk between hippo signalling and miRNAs in tumour progression. FEBS J. 2017;284(7):1045-55.

18. Zanconato F, Cordenonsi M, Piccolo S. YAP/TAZ at the roots of Cancer. Cancer Cell. 2016;29(6):783-803.

19. Dong J, Feldmann G, Huang J, Wu S, Zhang N, Comerford SA, Gayyed MF, Anders RA, Maitra A, Pan D. Elucidation of a universal size-control mechanism in Drosophila and mammals. Cell. 2007;130(6):1120-33.

20. Zhou D, Zhang Y, Wu H, Barry E, Yin Y, Lawrence E, Dawson D, Willis JE, Markowitz SD, Camargo FD, et al. Mst1 and Mst2 protein kinases restrain intestinal stem cell proliferation and colonic tumorigenesis by inhibition of yes-associated protein (yap) overabundance. Proc Natl Acad Sci U S A. 2011; 108(49):E1312-20.

21. Kalluri R, Weinberg RA. The basics of epithelial-mesenchymal transition. J Clin Invest. 2009;119(6):1420-8

22. Ye X, Weinberg RA. Epithelial-mesenchymal plasticity: a central regulator of Cancer progression. Trends Cell Biol. 2015;25(11):675-86.

23. Lei QY, Zhang H, Zhao B, Zha ZY, Bai F, Pei XH, Zhao S, Xiong Y, Guan KL. TAZ promotes cell proliferation and epithelial-mesenchymal transition and is inhibited by the hippo pathway. Mol Cell Biol. 2008;28(7):2426-36.

24. Overholtzer M, Zhang J, Smolen GA, Muir B, Li W, Sgroi DC, Deng CX, Brugge JS, Haber DA. Transforming properties of YAP, a candidate oncogene on the chromosome 11q22 amplicon. Proc Natl Acad Sci U S A. 2006;103(33):12405-10.

25. Lee DG, Kim HS, Lee YS, Kim S, Cha SY, Ota I, Kim NH, Cha YH, Yang DH, Lee Y, et al. Helicobacter pylori CagA promotes snail-mediated epithelialmesenchymal transition by reducing GSK-3 activity. Nat Commun. 2014;5:4423.

26. Jiao S, Wang H, Shi Z, Dong A, Zhang W, Song X, He F, Wang Y, Zhang Z, Wang $W$, et al. A peptide mimicking VGLL4 function acts as a YAP antagonist therapy against gastric cancer. Cancer Cell. 2014;25(2):166-80.

27. Ge Z. And D.E. Taylor, H. pylori DNA transformation by natural competence and electroporation. Methods Mol Med. 1997;8:145-52.

28. Li NS, Zou JR, Lin H, Ke R, He XL, Xiao L, Huang D, Luo L, Lv N, Luo Z. LKB1/ AMPK inhibits TGF-beta1 production and the TGF-beta signaling pathway in breast cancer cells. Tumour Biol. 2016;37(6):8249-58.

29. Xie C, Xu LY, Yang Z, Cao XM, Li W, Lu NH. Expression of gammaH2AX in various gastric pathologies and its association with helicobacter pylori infection. Oncol Lett. 2014;7(1):159-63.

30. Yang Z, Xie C, Xu W, Liu G, Cao X, Li W, Chen J, Zhu Y, Luo S, Luo Z, et al. Phosphorylation and inactivation of PTEN at residues Ser380/Thr382/383 induced by helicobacter pylori promotes gastric epithelial cell survival through PI3K/Akt pathway. Oncotarget. 2015;6(31):31916-26.

31. Ohata H, Kitauchi S, Yoshimura N, Mugitani K, Iwane M, Nakamura H, Yoshikawa A, Yanaoka K, Arii K, Tamai H, et al. Progression of chronic atrophic gastritis associated with helicobacter pylori infection increases risk of gastric cancer. Int J Cancer. 2004;109(1):138-43.

32. Lamar JM, Stern P, Liu H, Schindler JW, Jiang ZG, Hynes RO. The hippo pathway target, YAP, promotes metastasis through its TEAD-interaction domain. Proc Natl Acad Sci U S A. 2012;109(37):E2441-50.

33. Fock KM, Ang TL. Epidemiology of helicobacter pylori infection and gastric cancer in Asia. J Gastroenterol Hepatol. 2010;25(3):479-86.

34. Barrozo RM, Cooke CL, Hansen LM, Lam AM, Gaddy JA, Johnson EM, Cariaga TA, Suarez G, Peek RM Jr, Cover TL, et al. Functional plasticity in the type IV secretion system of helicobacter pylori. PLoS Pathog. 2013;9(2):e1003189.

35. Jang S, Su H, Blum FC, Bae S, Choi YH, Kim A, Hong YA, Kim J, Kim JH, Gunawardhana N, et al. Dynamic Expansion and Contraction of cagA Copy Number in Helicobacter pylori Impact Development of Gastric Disease. MBio. 2017:8(1).

36. Liu-Chittenden Y, Huang B, Shim JS, Chen Q, Lee SJ, Anders RA, Liu JO, Pan D. Genetic and pharmacological disruption of the TEAD-YAP complex suppresses the oncogenic activity of YAP. Genes Dev. 2012;26(12):1300-5.

37. Loh JT, Shaffer CL, Piazuelo MB, Bravo LE, McClain MS, Correa P, Cover TL. Analysis of cagA in helicobacter pylori strains from Colombian populations with contrasting gastric cancer risk reveals a biomarker for disease severity. Cancer Epidemiol Biomark Prev. 2011;20(10):2237-49.

38. Naumann M, Sokolova O, Tegtmeyer N, Backert S. Helicobacter pylori: a paradigm pathogen for subverting host cell signal transmission. Trends Microbiol. 2017;25(4):316-28.
39. Barranco SC, Townsend CM Jr, Casartelli C, Macik BG, Burger NL, Boerwinkle WR, Gourley WK. Establishment and characterization of an in vitro model system for human adenocarcinoma of the stomach. Cancer Res. 1983;43(4):1703-9.

40. Lamouille S, Xu J, Derynck R. Molecular mechanisms of epithelialmesenchymal transition. Nat Rev Mol Cell Biol. 2014;15(3):178-96.

41. Wang Y, Pan P, Wang Z, Zhang Y, Xie P, Geng D, Jiang Y, Yu R, Zhou X. beta-catenin-mediated YAP signaling promotes human glioma growth, 2017. J Exp Clin Cancer Res. $(36,1): 136$.

42. Mo JS, Meng Z, Kim YC, Park HW, Hansen CG, Kim S, Lim DS, Guan KL. Cellular energy stress induces AMPK-mediated regulation of YAP and the hippo pathway. Nat Cell Biol. 2015;17(4):500-10.

\section{Ready to submit your research? Choose BMC and benefit from:}

- fast, convenient online submission

- thorough peer review by experienced researchers in your field

- rapid publication on acceptance

- support for research data, including large and complex data types

- gold Open Access which fosters wider collaboration and increased citations

- maximum visibility for your research: over $100 \mathrm{M}$ website views per year

At BMC, research is always in progress.

Learn more biomedcentral.com/submissions 